Further Section

Cerebrovasc Dis 1997;7:1

\title{
Message from the Editors
}

\begin{tabular}{|l|l|l|}
\hline J. & & Bogousslavsky \\
\hline M. & Hennerici \\
\hline
\end{tabular}

\section{Cerebrovascular Diseases}

, Lausanne, Mannheim 


\section{Message from the Editors}

We would like to thank Drs. J. Biller (Indianapolis, Ind.), and A. Hakim (Ottawa) who leave the Advisory Editorial Board within the frame of our rotating policy. Drs. P. Amarenco (Paris), M.D. Ginsberg (Miami, Fla.), J. Grotta (Houston, Tex.), GJ. Hankey (Perth), M.E. Moseley (Stanford, Calif.), W. Sandmann (Diisseldorf), A. Schwartz (Mannheim), E. Diez-Tejedor (Madrid), and J. Theron (Caen) are joining the board and we would like to welcome them. We also wish to thank all ad hoc reviewers in 1996, whose help allowed to maintain the highest scientific standards for the journal.
N. Aebischer, Lausanne
P. Amarenco, Paris
H. Barnett, London, Ont.
C. Bassetti, Lausanne
S. Blecic, Brussels
U. Bleyl, Mannheim
T. Brandt, Munich
T. Brott, Cincinnati, Ohio
M.M. Brown, London
J.L. Cummings, Los Angeles, Calif.
M. Daffertshofer, Mannheim
S. Davis, Parkville, Vic.
P. de Merloose, Geneva
N. de Tribolet, Lausanne
H.Ch. Diener, Essen
$|S| \& E a s t o n$, Providence, R.L
W. Enzensberger,firankfurt
G. Ertl, Mannheim
K. Fassbender, Mannheim
F. Fazekas, Graz
H J. Freund, Diisseldorf
D. Georgiadis, Minister
M. Giroud, Dijon 
J. Grotta, Houston, Tex. V. Hachinski, London, Ont. V. Henn, Zurich I. Heuser, Mannheim V. Homberg, Diisseldorf C. Kase, Boston, Mass. P.J. Koudstaal, Rotterdam P. Langhorne, Edinburgh K. Lees, Glasgow F. Levi, Lausanne J. Lodder, Maastricht P. Maeder, Lausanne P. Magistretti, Lausanne H. Markus, London F. Mauguiere, Lyon C.H. Millikan, Toledo P. Moore, Detroit, Mich. T. Moulin, Besancon N. Murray, London J.P. Neau, Poitiers N. Nighoghossian, Lyon S. Oppenheimer, Baltimore T. Pinho e Melo, Lisbon 

K. Poeck, Aachen
G. Rancurel, Paris
W. Rautenberg, Diisseldorf
S. Ricci, Padova
E.B. Ringelstein, Minister
A. Ropper, Boston, Mass.
J. Rother, Jena
R. Sacco, New York, N.Y.
P. Sandercock, Edinburgh
A. Schwartz, Mannheim
D. Seidel, Munich
W. Steinke, Mannheim
M. Sturzenegger, Bern
P. Touboul, Paris
A. Valavanis, Zurich
G. van Melle, Lausanne
G. von Cramon, Leipzig
B. Widder, Ulm
P. Wolf, Boston, Mass. F. Yatsu, Houston, Tex. Y. Yonekawa, Zurich

\section{KARGER}

E-Mail karger@karger.ch Fax+4161 3061234

http://www.karger.ch 
